
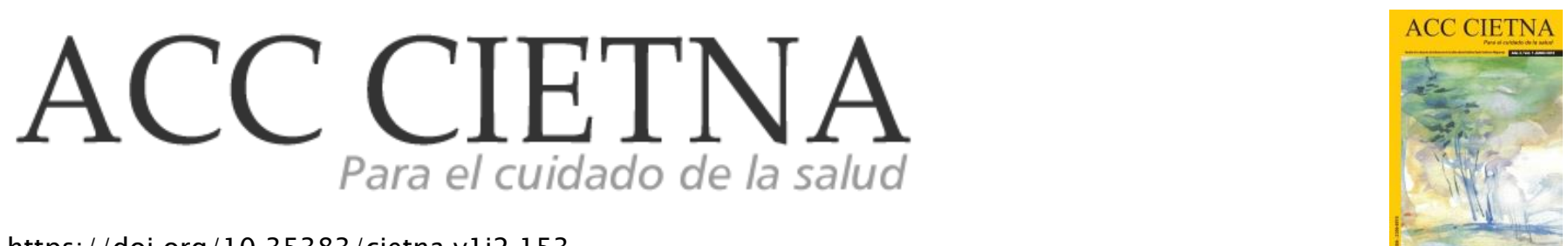

https://doi.org/10.35383/cietna.v1 i2.153

\title{
Accidentes de trabajo y elementos de protección personal en trabajadores de limpieza pública del distrito de José Leonardo Ortiz - Chiclayo, Perú
}

\author{
Macalopú Torres Sandra Inés ${ }^{1}$, Guzmán Tello Socorro ${ }^{2}$
}

INFORMACIÓN DEL ARTÍCULO
Historia del artículo:
Recibido el 3 de marzo de 2016
Aceptado el 5 de julio de 2016

Palabras claves:

Accidentes de trabajo

Elementos de protección personal

Trabajador de limpieza

Enfermería

\section{RESUMEN}

La presente investigación es de tipo cuantitativa, correlacional, descriptiva y transversal, la población fue de 220 personas y una muestra de 132 trabajadores de limpieza pública (solo en las labores de barrido de calles, avenidas y jardines) se utilizó como el instrumento para la recolección de datos fue una encuesta sobre la casuística de accidentes estructurada en ocho preguntas cerradas. Esta investigación es importante porque involucra al trabajador de limpieza, un ser vulnerable ante la sociedad, y la enfermería es la ciencia que ayudará a cuidar al trabajador de los peligros que se presenten en su trabajo, mediante la prevención y promoción de la salud, es así que formulamos como objetivo general: Conocer la relación que existe entre los accidentes de trabajo y el uso de los elementos de protección personal en el trabajador de limpieza pública de la Municipalidad de José Leonardo Ortiz-2012. El análisis estadístico se realizó con el Programa SPSS, Versión 18; se elaboraron tablas unidimensionales y bidimensionales de frecuencia, se aplicó la prueba Chi Cuadrado para comprobar la relación entre las variables. La significancia utilizada fue $8=0.05$. Así mismo, en los resultados se encontró que el $81.8 \%$ han sufrido algún accidente laboral porque no ha usado los elementos de protección personal. Un $90.9 \%$ de trabajadores de limpieza han sufrido algún accidente laboral y con respecto al uso de elementos de protección personal un $87.9 \%$ de trabajadores municipales no usan elementos de protección personal, llegando a la conclusión que existe relación entre los accidentes laborales y el uso de los Elementos de

\footnotetext{
${ }^{1}$ Licenciada en Enfermería. Enfermera con trabajo independiente, Chiclayo, Perú. Email: macalopu@gmail.com

2 Docente de la Escuela de Enfermería de la Universidad Católica Santo Toribio de Mogrovejo, Chiclayo, Perú. Email: 
Protección Personal en el trabajador de limpieza pública del distrito de José Leonardo Ortiz, el cual estuvo representado por un $81.1 \%$ de la muestra que sufrió algún accidente por no usar los elementos de protección personal.

Accidents and personal protection elements in workers public cleaning from district José Leonardo Ortiz - Chiclayo, Perú

ABSTRACT

Keywords:

Accidents at work

Personal protection

Janitor

Nurse
This research is quantitative , correlational , descriptive, cross-sectional , population was 220 people and a simple of 132 workers only included public sanitation in the work sweeping streets, avenues and gardens, which is a population-based sample ; the instrument used for data collection was a survey on the casuistry of accidents comprised eight closed questions. This research is important because it involves cleaning worker, be vulnerable in society, and nursing is the science that help care workers of the dangers that arise at work through prevention and health promotion, as formulated overall objective: To determine the relationship between occupational accidents personal protection public worker cleaning the Municipality of Jose Leonardo Ortiz , 2012 . Statistical analysis was performed with the SPSS program, version 18, one and two dimensional frequency tables were produced, the Chi Square test was applied to test the relationship between variables. Significance $8=0.05$ was used. Likewise, the results found that $81.8 \%$ have had a work accident that has not used the personal protection. A $90.9 \%$ of janitors have had a work accident and regarding the use of personal protective equipment $87.9 \%$ of municipal workers use personal protective equipment, concluding that there is a relationship between accidents and the use of elements of Personal protection in the public sanitation worker district José Leonardo Ortiz, who was represented by $81.1 \%$ of the sample suffered an accident by not using personal protective equipment.

\section{Introducción}

Habitualmente se ha visto a la mayoría de centros de laborales en diferentes actividades ocupacionales sufrir accidentes en su centro de labores, esto es, sucesos repentinos y prevenibles que sobrevienen por causa o con ocasión del trabajo, y que producen en el trabajador una lesión orgánica, una perturbación funcional, una invalidez o muerte. ${ }^{1}$
Al respecto la Organización Internacional del Trabajo (OIT) reporta estadísticas anuales de 120 millones de accidentes de trabajo en el mundo; de estos, 210000 se registran como defunciones. A la vez, se han contabilizado 270 millones de accidentes laborales con 2 millones de muertes relacionadas con el trabajo y pérdidas del $4 \%$ del Producto Bruto Interno mundial por accidentes y enfermedades ocupacionales. Por su parte, en Latinoamérica y El Caribe han ocurrido 30 millones de accidentes (1-5\%), 40000 de ellos fueron 
mortales. ${ }^{2}$ En cuanto a los datos estadísticos en Chile por accidentes de trabajos catalogados como accidentes fatales, se reportan 28 casos por golpe (18.5\%) y por aplastamiento 26 casos (17.2\%) en el año 2007 y 2008. En cuanto a servicios comunales y sociales que involucra la limpieza pública, se obtuvieron 26 casos (15.9\%). ${ }^{3}$ Con respecto a datos estadísticos en el Perú, se reporta una clasificación por el tipo de accidente: accidentes leves (37.93\%), accidentes incapacitantes (59.05\%), y accidente mortal (3.03\%). 4

Para el presente estudio se tomó en cuenta a los trabajadores de limpieza pública del distrito José Leonardo Ortiz, el cual cuenta con una población de 165483 habitantes y con 220 personas que trabajan en limpieza pública: barredores de calles, avenidas y jardines, recolectores de basura y los choferes de los camiones de basura.

En las calles del distrito se observa con mucha frecuencia a los trabajadores de limpieza de José Leonardo Ortiz sustituyendo elementos de protección personal (EPP) por materiales simples para desempeñar distintas labores como, por ejemplo, la utilización de franelas para cubrir la boca y nariz para protegerse del polvo al momento de barrer, zapatillas de lona; asimismo realizan largas caminatas en el barrido de calles, ya que muchas veces son más de diez cuadras las que se encuentran con montículos de basura donde se observa su manipulación sin ninguna protección o utilización de bolsas plásticas para recogerlas; utilizan ropa común para desempeñar sus funciones generando posibles lesiones $y$ accidentes laborales.

Al interactuar con unos de los trabajadores de tienen que barrer. En el trayecto de su labor se limpieza, estos muestran desinterés y desconocimiento sobre los accidentes de trabajo. Mientras se les hablaba seguían trabajando, no rol que cumple la enfermera ocupacional en el prestaban mucha atención. He ahí la importante área preventiva promocional, cuya misión es la educación e información adecuada, precisa y óptima para el entendimiento y aplicación por parte de la persona. Además, se menciona que las horas de trabajo son 8 , de lunes a sábado en específicamente se trabaja una semana en la horario semanal de mañana y tarde, mañana y la siguiente en la tarde. Su horario de entrada es a las 7:30 de la mañana hasta la 3:00 de sus alimentos.

Por lo expuesto, se plantea el siguiente objetivo la tarde, dando un tiempo de una hora para ingerir general: Conocer cuál es la relación que existe entre accidentes laborales y el uso de elementos de protección personal en el trabajador de limpieza pública del distrito de José Leonardo Ortiz, y como objetivos específicos: Analizar la incidencia de accidentes de trabajo en los trabajadores de limpieza pública del distrito José Leonardo Ortiz y determinar el uso de los elementos de protección personal en los trabajadores de limpieza.

\section{Metodología}

La investigación fue de tipo cuantitativa, correlacional, descriptiva y transversal. La población fue de 220 personas y la muestra, de 132 trabajadores de limpieza pública, que realizan sus labores de barrido en calles, avenidas y jardines; el instrumento que se utilizó para la recolección de datos fue una encuesta sobre la casuística de accidentes, conformada por ocho preguntas cerradas, las cuales permitieron describir mejor la información en cuanto a accidentes laborales y el uso de los elementos de protección personal.

\section{Resultados, análisis y discusión}

Tabla 1. Relación entre los accidentes laborales y el uso de los elementos de protección personal en los trabajadores de limpieza pública del distrito José Leonardo Ortiz. 


\begin{tabular}{lllllll}
\hline \multirow{2}{*}{ Accidentes laborales } & \multicolumn{6}{l}{ Uso de Elementos de Protección personal } \\
\cline { 2 - 6 } & $\mathrm{N} 0$ & \multicolumn{5}{c}{$\mathrm{Si}$} \\
\cline { 2 - 6 } & $\mathrm{n}$ & $\%$ & $\mathrm{n}$ & $\%$ & $\mathrm{n}$ & $\%$ \\
\hline $\mathrm{No}$ & 8 & $6.1 \%$ & 4 & $3.0 \%$ & 12 & $9.1 \%$ \\
$\mathrm{Si}$ & 108 & $81.8 \%$ & 12 & $9.1 \%$ & 120 & $90.9 \%$ \\
\hline Total & 116 & $87.9 \%$ & 16 & $12.1 \%$ & 132 & $100.0 \%$ \\
\hline Fuente: Encuesta aplicada a los trabajadores de limpieza pública del distrito de José Leonardo Ortiz.
\end{tabular}

Encontramos que el $81.8 \%$ de trabajadores de limpieza que ha sufrido algún accidente laboral no ha usado elementos de protección personal. Así mismo, un $90.9 \%$ de trabajadores de limpieza ha sufrido algún accidente laboral y con respecto al uso de elementos de protección personal un $87.9 \%$ de trabajadores municipales no usa elementos de protección personal., como se puede apreciar en la Tabla 2.

Tabla 2. Uso de los elementos de protección personal de los trabajadores de limpieza de la Municipalidad José Leonardo Ortiz.

\begin{tabular}{lll}
\hline Uso de los EPP & N. & $\%$ \\
\hline Si & 16 & 12.1 \\
No & 116 & 87.9 \\
\hline Total & 132 & 100.00 \\
\hline Fuente: Encuesta aplicada a los trabajadores de limpieza publica del distrito de José Leonardo Ortiz.
\end{tabular}

El $12.1 \%$ de los trabajadores de limpieza de la Municipalidad José Leonardo Ortiz usa los elementos de protección personal, y el $87.9 \%$ no lo hace.

Tabla 3. Tipo de accidentes laborales sufridos por parte de los trabajadores de limpieza de la Municipalidad José Leonardo Ortiz.

\begin{tabular}{lllll}
\hline \multirow{2}{*}{ Accidentes } & \multicolumn{3}{l}{ Sí } & \multicolumn{3}{l}{ No } \\
\cline { 2 - 5 } & $\mathrm{N} .^{0}$ & $\%$ & $\mathrm{~N}^{0}{ }^{\circ}$ & $\%$ \\
\hline Corte superficial & 72 & 54.5 & 60 & 45.5 \\
Corte profundo & 15 & 11.4 & 117 & 88.6 \\
Pinchazo & 75 & 56.8 & 57 & 43.2 \\
Golpes & 62 & 47.0 & 70 & 53.0 \\
Aplastamiento por contenedores & 29 & 22.0 & 103 & 78.0 \\
Caidas, resbalones & 74 & 56.1 & 58 & 43.9 \\
Sobreesfuerzo muscular & 48 & 36.4 & 84 & 63.9 \\
Atropello vehicular & 10 & 7.6 & 122 & 92.4 \\
\hline
\end{tabular}

Fuente: Encuesta aplicada a los trabajadores de limpieza pública del distrito de José Leonardo Ortiz.

El $54.5 \%$ de los trabajadores de limpieza de la Municipalidad José Leonardo Ortiz ha sufrido de cortes superficiales, el $11.4 \%$ de cortes profundos, el $56.8 \%$ de pinchazos, el $47 \%$ de golpes, el $22 \%$ de aplastamiento por contenedores, el $56.1 \%$ de caídas y resbalones, el $36.4 \%$ ha padecido sobreesfuerzo muscular y el $7.6 \%$ de atropellamiento vehicular.

En el mundo laboral el trabajo es fuente de salud pues mediante ello las personas logran acceder a una serie de cuestiones favorables para la mantención de un estado de bienestar; una comunidad o un país mejoran el nivel de salud de la población cuando aseguran que todas las personas económicamente activas pueden acceder a un empleo que satisfaga no solo sus necesidades económicas básicas, sino que también los otros aspectos positivos del trabajo, los cuales son: salario, actividad física y mental, contacto social, producción de bienes y servicios. ${ }^{5}$ Se considera al trabajo como todo esfuerzo físico o mental destinado a la producción de los bienes necesarios para nuestra supervivencia; 6 así mismo Mazzáfero ${ }^{7}$ menciona que el trabajo en la actualidad resulta imprescindible para una vida saludable, tanto por la retribución económica que comporta, como por el hecho social que contribuye a la realización de la persona y al progreso de la comunidad. Así mismo, es importante tener en cuenta que el ambiente de trabajo es el medio más exigente en el que se mueve el hombre en función de la intensidad de exposiciones ya sean físicas, químicas, biológicas, ergonómicas o psicológicas, de modo que sirve de sistema de alerta e incluso de modelo para actividades preventivas que posteriormente incluyen al resto de la población, definiendo así a los trabajadores como grupos en riesgo. $^{7}$

Dentro de este ámbito es importante destacar que una de las labores importantes es la limpieza pública, que se entiende como la preservación de las condiciones higiénico - sanitarias en los lugares públicos, desde la recolección hasta el destino final de los residuos sólidos. Esta labor es ejercida por los trabajadores de limpieza, encargadas de mantener las calles y jardines de la 
ciudad limpias y libre de contaminación. ${ }^{8}$ Sin embargo, estas personas, habitualmente se encuentran expuestas a distintos tipos de accidentes laborales como: caídas, resbalones, pinchazos, cortes, sobreesfuerzos, atropello vehicular, etc., los cuales pueden provocar daños en su salud, además epidemiológicamente se podría considerar como un grupo vulnerable frente a los accidentes de trabajo. ${ }^{9}$

Según estudios realizados en Chile (2007-2008), se han reportado 26 casos de accidentes de trabajo dando como resultado el 15.9\%; de igual manera en el Perú en el año 2011 se notificaron 301 accidentes de trabajo, de los cuales el $6.36 \%$ corresponde al servicio de limpieza pública. 4 En la presente investigación realizada en el distrito de José Leonardo Ortiz se encontró que los trabajadores de limpieza pública que han sufrido algún tipo de accidente laboral está representado por un $90.9 \%$, de los cuales el $81.1 \%$ se accidentó por no usar los elementos de protección personal. De este modo, Ruiz 6 menciona que un accidente de trabajo es un suceso súbito, inesperado y no deseado que puede causar daño al trabajador produciendo perturbación funcional, invalidez o la muerte; así mismo un accidente de trabajo se produce durante la ejecución de las órdenes de un empleador o durante la ejecución de una labor bajo su autoridad, aun fuera del lugar de trabajo. ${ }^{8}$

En el artículo 14 de la Ley de Prevención de Riesgos Laborales se señala que los trabajadores tienen derecho a una protección eficaz en materia de seguridad y salud en el trabajo. Este derecho supone la existencia de un correlativo deber del empresario sobre la protección de los trabajadores frente a los riesgos laborales, cuyo incumplimiento se considera infracción grave. 10 Por lo tanto, teniendo en cuenta el derecho a la protección eficaz en cuanto a la seguridad esta muchas veces se descuida, evidenciándose en la deficiente demanda de capacitaciones, asesorías, campañas educativas, para aquellas personas que ejercen la labor de limpieza pública del distrito de José Leonardo Ortiz, de la misma manera es necesario considerar que además de las capacitaciones es indispensable para la seguridad del trabajador enfocarse sobre la importancia que tiene la renovación frecuente de los elementos de protección personal; en tal sentido se encontró que de los trabajadores encuestados el $96.2 \%$, afirman que reciben cada año los elementos de protección personal y el $3.8 \%$ cada seis meses.

Como señala, Landauro'1 respecto a los EPP, se renovará cada vez que se encuentre desgastado, dañado o deteriorado, respetando el tiempo promedio de recambio, es decir, en cuanto al uso de mascarillas simples se renueva en un lapso de dos semanas, la ropa de trabajo se renueva en un año, el uso de cambiadas cuando la cobertura no proporcione guantes en seis meses, las botas de seguridad serán suficiente protección al pie. La suela puede cambiarse mientras la cobertura se encuentre en buenas condiciones. Generalmente muchas personas en este ámbito laboral no usan de forma correcta los considera importante capacitar al personal en cuatro elementos de protección personal, por ello se a la importancia del uso de los elementos de protección personal. Al respecto, en el estudio se encontró que el $53.8 \%$ afirmaron que nunca han tenido una capacitación sobre temas relacionados con su seguridad y salud, esto hace suponer que los trabajadores que no usan los EPP, es porque capacitaciones en la formación del personal ocupan desconocen su importancia y los riesgos de no usarlos.

Por consiguiente, Mazzáfero 7 afirma que las un lugar muy importante en lo que se afirma a prevención de accidentes y enfermedades, el ordenamiento legal considera básico que las partes interesadas (empresas, asociaciones, empleadores y sindicatos) participen en la elaboración de programas y campañas educativas e informativas sobre prevención (art. $5^{\circ}$, inc.m, Ley 19.587 y 214 , dec. Regl.), en tanto el empleador debe realizar en el ámbito laboral un plan anual de capacitación por medio de curso, seminarios, etc. para de esta manera mantener un espíritu alerta en el personal respecto del riesgo de trabajo. 
Además, en la protección del trabajador de limpieza influyen elementos de protección personal, pues en el artículo 7 de la Ley de Seguridad e Higiene en el Trabajo; las reglamentaciones de las condiciones de seguridad deberán considerar primordialmente: equipos de protección personal de los trabajadores; prevención de accidentes del trabajo y enfermedades laborales. ${ }^{7}$ En relación a los elementos de protección personal, se observó que en la Tabla 2 un $87.9 \%$ de los trabajadores no usan los elementos de protección personal, y el $12.1 \%$ lo utilizan correctamente. Sin embargo, en la investigación realizada por las autoras Bastidas $\mathrm{E}$. y Rodríguez L. (Ibarra-Ecuador), se obtuvo como resultados que un $18.2 \%$ de los trabajadores de limpieza pública utilizan de la mejor manera los elementos de protección personal, pero un porcentaje inferior del $12.1 \%$ no se protege con ningún elemento de protección personal. 12

Al analizar estas realidades, en el distrito de José Leonardo Ortiz solo hay un $12.1 \%$ de los trabajadores que sí utilizan correctamente los elementos de protección personal lo que devela que existe una falta de sensibilización, de educación, capacitación e indumentaria para el trabajador; en cambio en la investigación realizada en Ecuador se demuestra que existe una mejor cultura de prevención de accidentes laborales, gracias al uso de los elementos de protección personal como son: botas, guantes y overol. Al respecto, Valencia ${ }^{13}$ considera que un elemento de protección personal es todo equipo, aparato o dispositivo especialmente proyectado y fabricado para preservar el cuerpo humano, en todo o en parte, de riesgos específicos de accidentes de trabajo; así mismo Mazzáfero ${ }^{7}$ menciona que la protección personal está constituida por aquellos elementos que emplea el trabajador con el objeto de disminuir o evitar lesiones corporales, además de ello considera que la protección personal es la última barrera entre el hombre y el riesgo.
Por lo tanto, los elementos de protección personal son importantes en todo momento durante el trabajo diario, pues con el uso correcto de los dispositivos se puede prevenir muchos accidentes laborales. Se afirma que se encontró relación entre los accidentes laborales y el uso de los elementos de protección personal en el trabajador de limpieza del distrito de José Leonardo Ortiz, demostrado en la Tabla $l$ donde se evidencia que el $81.1 \%$ de los trabajadores de limpieza ha sufrido algún accidente laboral debido al no uso de los elementos de protección personal, contra el 9.1\% de trabajadores que ha sufrido accidente laboral haciendo uso de elementos de protección personal. Si bien es cierto, el autor Mazzáfero refiere que un accidente de trabajo es la ruptura en el equilibrio necesario entre el hombre y sus condiciones de trabajo. Es un evento no planeado, dado en relación compleja del individuo y su ambiente de actividad productiva que da como resultado un deterioro de esa relación. Además, representa un daño físico y un sufrimiento para el (pérdidas de tiempo, y productividad, pérdida de trabajador y daños para el proceso productivo materiales, ruptura de equipos, etc.). ${ }^{7}$

Al mismo tiempo, un accidente de trabajo es siempre el resultado de la interacción de múltiples factores entre los que se destacan los propios del ambiente de trabajo (condiciones físicas ambientales de trabajo, equipos de trabajo, organización de trabajo, ritmos de trabajo, relaciones de trabajo, etc.).6,7 Alrededor de estos conceptos de acuerdo al análisis de la problemática se encontró en el estudio que los tipos de accidentes laborales más frecuentes en los trabajadores de limpieza pública de José Leonardo Ortiz, según la clasificación, son los leves, como: cortes superficiales (54.5\%), cortes profundos (11.4\%), pinchazos (56.8\%), caídas y resbalones (56.1\%). Al respecto Rodríguez, 14 en su trabajo de investigación titulado "Riesgos Ocupacionales y Accidentes laborales en trabajadores de distritos de la región Amazonas, mencionó menores incidencias: como caídas (5.8\%), heridas cortantes (4.4\%) y atropello 
vehicular $(3,7 \%)$. Lo que hace suponer que estos trabajadores de limpieza pública se encuentran más instruidos, aunque tal vez no en su totalidad; y que además actúan de manera más responsable en comparación de los trabajadores del distrito de José Leonardo Ortiz, que presentan elevados índices de accidentes laborales, superiores a la realidad de los trabajadores de Amazonas.

Visto de esta forma, los accidentes leves son sucesos resultantes de una lesión que luego de la evaluación médica correspondiente pueden generar en el accidentado un descanso breve (retorno máximo: al día siguiente a sus labores habituales).

Evidentemente estos accidentes pueden generar cortes, que son heridas en la piel provocadas por objetos afilados; pinchazos, que se define como la agresión brusca y repentina producida por un objeto puntiagudo; y los golpes, que es aquel movimiento rápido y brusco producido por un suceso inesperado. 15,16

Por otro lado, debe señalarse que un accidente incapacitante es un suceso resultante en lesiones que, luego de la evaluación médica correspondiente, da lugar a descanso médico y tratamiento a partir del día siguiente de sucedido el accidente. Un accidente mortal es aquel suceso resultante en lesión que produce la muerte del trabajador al margen del tiempo transcurrido entre la fecha de accidente y la muerte. Para efecto de la estadística se debe considerar la fecha del deceso. ${ }^{15}$ Al ubicarse en la realidad de José Leonardo Ortiz mayormente se encontraron accidentes leves en lo que se refiere a los cortes por vidrios que son más comunes en la labor diaria, los pinchazos por agujas, clavos, alambres, debido al inadecuado uso de los guantes, y las caídas o resbalones por el uso de botas inadecuadas.

Es importante conocer que una de las medidas preventivas es la utilización de los EPP definidos como todo equipo, aparato o dispositivo especialmente proyectado y fabricados para preservar el cuerpo humano, en todo o en parte, de riesgos específicos de accidentes del trabajo o enfermedades profesionales.13,17 Esta relación implica necesariamente que su uso es indispensable para el trabajador de limpieza pública durante sus labores diarias, esto no quiere decir que el trabajador se encuentra libre de sufrir algún accidente, el peligro está siempre presente, la función que cumplen los elementos de protección personal son reducir significativamente el impacto de algún riesgo y proteger a la persona. ${ }^{13}$ En tal sentido, en el estudio se encontró que los guantes de jebe son utilizados algunas veces en un 53\%; las botas, en un $67.4 \%$; en lo que se refiere al uso de overol nunca lo utilizan en un $91.7 \%$; por otra parte en cuanto al uso de mascarilla de tela hay un $43.2 \%$ que lo utiliza frecuentemente y respecto a las mascarillas descartables un $81.1 \%$ nunca lo utilizan.

Al respecto, Bastidas y Rodríguez ${ }^{12}$ en su trabajo de investigación titulado Enfermedades prevalentes en los trabajadores municipales de recolección de basura de la ciudad de Ibarra, encontraron que el $64 \%$ de los trabajadores solo utiliza overol y guantes, en cambio un $18 \%$ utiliza de mejor manera las normas de bioseguridad ya que usan guantes, overol y botas, pero un porcentaje inferior de $12.1 \%$ no se protege con ningún implemento, excepto el overol. En relación a los trabajadores de limpieza pública del distrito de José Leonardo Ortiz, se obtuvo que un $87.9 \%$ de los trabajadores no utilizan correctamente dichos dispositivos que son esenciales para cuidar su vida y salud, e igualmente no cumplen con la Ley de Prevención de Riesgos Laborales y por ende .infringen normas Al mismo tiempo, de acuerdo a la investigación realizada en el distrito de IbarraEcuador, 12 se encontraron porcentajes inferiores en lo que se refiere al no uso de los elementos de protección personal, esto quiere decir que hay más coordinación, interacción entre el empleador y trabajador para identificar las necesidades en el trabajo diario. 
En consecuencia, es importante reconocer que la Ley de Prevención de Riesgos Laborales define a la prevención como el conjunto de medidas adoptadas o previstas en todas las fases de actividades en un ambiente con el fin de evitar o disminuir los riesgos derivados del trabajo. (art.4.1). 10 Los EPP vienen a ser esencialmente útiles porque abarcan una de las medidas preventivas, al definirse como todo equipo, aparato o dispositivo especialmente proyectado y fabricado para preservar el cuerpo humano, en todo o en parte, de riesgos específicos de accidentes del trabajo. ${ }^{7}$ Es necesario tener en cuenta que la misión de los EPP es impedir una lesión o un daño al trabajador, para ello el equipo debe cumplir con estas mínimas características: ser cómodos y estéticos, ser adecuados al riesgo, de uso individual, de conservación adecuada (deben seguirse las recomendaciones del fabricante); el equipo debe estar homologado por los organismos habilitados; además de su funcionalidad, deben cuidarse otros aspectos como la conservación material, diseño de equipo, comodidad, etc. 7,18

Vinculando los conceptos, coincidimos con Ruiz, cuando menciona que los EPP son cualquier equipo destinado a ser llevado o sujetado por el trabajador para que lo proteja de uno o varios riesgos, que puedan amenazar su seguridad o su salud en el trabajo, así como cualquier complemento o accesorio destinado a tal fin. Está excluida de la definición la ropa de trabajo corriente, pero no la que ofrece protección frente a algún riesgo. 6 En líneas generales, de acuerdo a la realidad del personal de limpieza pública del distrito de José Leonardo Ortiz, es importante considerar que la enfermera es la profesional indicada para promover su salud y prevenir los daños de su entorno social y la encargada de, conllevar e al desarrollo de una cultura preventiva. En este estudio la Asociación Americana de Enfermeras de Salud Ocupacional (AAOHN) define la práctica de la enfermera en la salud ocupacional como: "La especialidad que provee y otorga servicios en el cuidado de la salud a los trabajadores. El ejercicio se enfoca a la promoción, protección y restauración de la salud de los trabajadores dentro del contexto de un ambiente de enfermería en la salud ocupacional es autónoma e trabajo seguro y saludable. La práctica de la independiente en la provisión de servicios de salud ocupacional. 5,18

En tal sentido el propósito de la enfermería en salud ocupacional es brindar atención de enfermería basada en modelos innovadores que consideren al trabajador como un ser bio-psicosocial, a su familia y a la sociedad en su conjunto. ${ }^{8}$ Las investigadoras acotan que dichos modelos además deben sustentarse en las necesidades del personal de limpieza pública. Y se respaldan en los resultados encontrados en la investigación: el $57.6 \%$ de los trabajadores considera que lo más importante es tener una atención de enfermería en el centro de trabajo y además de ello el $31.8 \%$ menciona que necesita una renovación frecuente de los elementos de protección personal. Cabe considerar que de acuerdo a la Ley de Prevención de Riesgos Laborales, artículo 4 y 5 , es importante proteger la vida, preservar y mantener la integridad psicofísica de los trabajadores y tener en cuenta la creación de servicios de higiene y seguridad en el trabajo, además sobre medicina del trabajo de carácter preventivo y asistencial. 10,19

Cabe resaltar que según la Ley de Prevención de Riesgos Laborales, con respecto a los Elementos de Protección Personal, todo trabajador debe utilizar los equipos de protección personal para mantener la prevención de accidentes del trabajo $y$ enfermedades ocupacionales. ${ }^{10}$ Quiere decir que los trabajadores están en todo el derecho de proteger su vida y su salud, obteniendo los equipos de protección personal los mismos que deben ser renovados cuando se desgasten o deterioren así se podrán evitar accidentes laborales.

\section{Conclusiones}

Arribamos a las siguientes conclusiones: 
Existe relación entre los accidentes laborales y el uso de los Elementos de Protección Personal en el trabajador de limpieza pública del distrito de José Leonardo Ortiz, esta estuvo representada por un 81.1 \% de la muestra que sufrió algún accidente por no usar los elementos de protección personal. Por consiguiente, se debe considerar a un EPP como uno de los dispositivos indispensables en la disminución de los accidentes laborales.

De acuerdo a la incidencia de los accidentes de trabajo, un $90.9 \%$ de los trabajadores ha sufrido algún tipo de accidente laboral, entre ellos se mencionan: los cortes superficiales, caídas, resbalones, pinchazos. Estos tienen un porcentaje considerable y muchas veces se ven desencadenados por la falta de conciencia del mismo trabajador, pues durante la entrevista se observó el poco interés por el cuidado de su salud, la poca o deficiente capacitación en el lugar donde laboran, como se constata en la encuesta realizada refieren que cada año reciben capacitaciones, y en lo que se refiere al uso de los EPP muchas veces no lo utilizan.

En lo que respecta a los elementos de protección personal en los trabajadores de limpieza pública del distrito de José Leonardo Ortiz, se obtuvo que un $12.1 \%$ de los trabajadores usan los EPP, y el $87.9 \%$ no lo utilizan. Se debe considerar que los trabajadores de limpieza pública están diariamente expuestos a múltiples riesgos laborales, por ello es indispensable el uso de los EPP, dichos dispositivos muchas veces ayudan a disminuir el riesgo al que está expuesto el trabajador y, por ende, proporcionan una barrera entre un determinado riesgo y la persona además de resguardar su integridad física del personal que labora.

\section{Bibliografía}

1. MINISTERIO DE SALUD. Dirección General de Salud Ambiental. 2005 [acceso 2 de Abril del
2012]. Albinagorta J, Tello J., Burga M, Fausto S, Bellido B, Ramírez $P$, et al. Manual de Salud Ocupacional.

Disponible en: http:/ / www.digesa.sld.pe/publicaciones/salud_ ocupacional.asp

2. Organización Internacional de Trabajo (OIT). La Seguridad en Cifras: Sugerencias para una cultura general en materia de seguridad en el trabajo. Ginebra; 2003

3. Organización Mundial de la Salud (OMS)/ Organización Internacional del Trabajo (OIT). Eje para la Acción Sindical: Conceptos básicos en Salud Laboral. Santiago, OIT; 2003. Salud y Trabajo aclarando los conceptos: Disponible en: http://oitchile.cl/pdf/publicaciones/ser/ser009.p df

4. Ministerio de Trabajo y promoción de empleo http://www.mintra.gob.pe/archivos/file/estadisti cas/anuario/ANUARIO_ESTADISTICO_2011.pdf

5. Rodríguez E, Enfermería en Salud Ocupacional. Ed. Lima-Perú: ASENSA; 1991

6. Ruiz C, García A, Delclós J, Benavides F. Salud Laboral. Conceptos y técnicas para la prevención de riesgos laborales. 3era Ed. Elsevier Masson. Barcelona 2006da

7. Mazzáfero E, Medicina en Salud Pública.2 ed. Buenos Aires: El Ateneo; 1999

8. Frías A. Salud Pública y educación para la salud. Masson. 2004

9. Hall J, Redding B. Enfermería en Salud Comunitaria: Un enfoque de Sistemas. Ed. PALTEX, 1990.

10. Ley de Prevención de Riesgos Laborales. Ley 54/2013. Disponible en: http://www.ugt.es/DatoBasico/prl08.pdf 
11. Landuro N. Procedimiento de Equipo de Protección Personal. 2006. Ed. Lima Airport Partners.

12. Rodríguez .L, Bastidas C. Enfermedades Prevalentes en los Trabajadores Municipales de Recolección de Basura de la Ciudad de Ibarra. Art.; nov.-oct. $2010 . \quad$ Disponible en: http://repositorio.utn.edu.ec/handle/ 123456789 1656

13. Valencia M. Manual de Seguridad, Salud Ocupacional y Ambiente para Contratistas. Universidad Nacional de Colombia. Mayo, 2008.

14. Rodríguez M, Pelaéz P. Riesgos ocupacionales y accidentes laborales en trabajadores de distritos de la región Amazonas. Rev. Enferm. Herediana; 1(2):87-92, jul.-dic. 2008. Disponible en: http://bases.bireme.br/cgi-

bin/wxislind.exe/iah/online/?lsisScript=iah/iah.xi $\mathrm{s} \& \mathrm{src}=$ google \&base $=$ LILACS\&lang $=$ p\&nextAction $=$ Ink\&exprSearch $=559028 \&$ indexSearch $=$ ID

15. Díaz R. Seguridad y salud en los regímenes laborales especiales. $1^{\circ}$ Ed. Lima-Perú Gaceta Jurídica 2011
16. Gallego A, Márquez A, Millán J, Monereo J, Moreno N, Vida R, et al. Manual para la formación en prevención de riesgos laborales. Lex Nova 4ed. España 2006

17. Instituto de Salud Pública de Chile. Instructivo Técnico. Listado Básico de Elementos de Protección (acceso 20 de Mayo del 2012) Departamento de Salud Ocupacional. Disponible en:

18. Instituto de Salud Pública de Chile. Instructivo Técnico: Listado Básico de de Elementos de Protección Personal. 2005. Disponible en: http://www.ispch.cl/salud_ocup/doc/listado_ba sico_epp.pdf

19. Juárez A, Hernández E. Intervenciones de enfermería en la salud en el trabajo. Rev Enferm Inst Mex Seguro Soc 2010; 18 (1): 23-29. Disponible en: http://www.medigraphic.com/ pdfs/enfermeriaimss/eim-2010/eim101 e.pdf 